\title{
O JUDÔ COMO FERRAMENTA DE SOCIALIZAÇÃO E PROMOÇÃO A SAÚDE PARA ADOLESCENTES COM TEA
}

\author{
${ }^{1}$ Ana Julia Ferreira Andrade ${ }^{2}$ Maria Beatriz Farias Duda ${ }^{3}$ Hildeberto Dutra Sobral
}

\section{RESUMO}

Introdução: O TEA é caracterizado como um transtorno complexo, de caráter mental e que prejudica o desenvolvimento neuro-motor do indivíduo, afetando a linguagem, interação social e cognição. $O$ judô se torna benéfico para o adolescente com TEA por se ter melhoras na coordenação motora, no domínio corporal e na socialização. Objetivo: Compreender de que forma a prática do Judô pode contribuir na melhora da coordenação, flexibilidade e qualidade de vida em adolescentes com TEA. Método: Trata-se de uma revisão integrativa da literatura realizada a partir das bases de dados: PubMed, Scielo, portal Biblioteca Virtual de Saúde - BVS, onde utilizou-se o operador booleano AND e os seguintes descritores: "Transtorno do Espectro Autista", "artes marciais", "Judo" em inglês e português. Como critério de inclusão: ensaios clínicos randomizados, nos idiomas inglês e português, publicado no período de 2016 a 2021 e que tinham como limite no estudo crianças e adolescentes (1019anos). Utilizaram-se como critérios de exclusão: estudo de casos, estudos com animais e os que não respondiam à pergunta norteadora. Os artigos foram selecionados conformerespondessem o objetivo da pesquisa pela análise dos resumos e leitura na íntegra. Resultados: Através das pesquisas foram observados pontos importantes em relação ao estudo de adolescentes com TEA., como o relato dos familiares, que afirmam que a interação social dos adolescentes após participarem das aulas é incentivada. Conclusão: Tendo em vista um aumento da população com TEA, surge à necessidade de estudos mais voltados a socialização e estímulos motores para evitar que esses jovens tenham sua qualidade de vida afetada. Contudo, foi chegado ao entendimento que crianças e adolescentes que frequentam locais onde o seu desenvolvimento cognitivo é estimulado tentem a ser mais sociáveis, adquirindo estímulos que podem reduzir os sintomas do TEA.

Palavras-chave: Transtorno do Espectro Autista, artes marciais e Judô.

\section{JUDÔ AS A TOOL FOR SOCIALIZATION AND HEALTH PROMOTION FOR TEENAGERS WITH} TEA

\begin{abstract}
Introduction: ASD is characterized as a complex mental disorder that impairs the individual's neuromotor development, thus affecting language, social interaction and cognition. Judo is beneficial for adolescents with ASD due to improvements in motor coordination, body control and socialization. Objective: To understand how the practice of Judo can contribute to improving coordination, flexibility and quality of life in adolescents with Autistic Spectrum Disorder. Method: This is an integrative literature review carried out from the following databases: PubMed, Scielo through the Virtual Health Library - VHL portal, where the Boolean operator AND was used and the following descriptors were used: " Disorder of the Autistic Spectrum", "martial arts", "Judo" in English and Portuguese. As inclusion criteria: randomized clinical trials, in English and Portuguese, published from 2016 to 2021 and which had children and adolescents (10-19 years old) as a limit in the study. Exclusion criteria were: case studies, studies with animals and those that did not answer the guiding question. The articles were selected as they answered the research objective by analyzing the abstracts and reading them in full. Results: Through the surveys, some important points were observed in relation to the study of adolescents with ASD, such as the report of family members, who claim that the adolescents' social interaction after participating in classes is encouraged. Conclusion: Given an increase in the population with ASD, there is a need for more studies focused on socialization and motor stimuli to prevent these young people from having their quality of life affected. However, it was understood that children and adolescents who attend places where their cognitive development is stimulated try to be more sociable, acquiring stimuli that can reduce ASD symptoms.
\end{abstract}

Keywords: Autistic Spectrum Disorder, martial arts and Judo. 


\section{Judô e socialização}

1. Estudante do Curso de Bacharel em Educação Física, Centro Universitário Mauricio de Nassau (UNINASSAU) - Campus Graças, Pernambuco, Brasil. 2.Estudante do Curso de Bacharel em Educação Física, Centro Universitário Mauricio de Nassau (UNINASSAU) - Campus Graças, Pernambuco, Brasil 3. Professor do Curso Bacharel em Educação Física, Centro Universitário Maurício de Nassau (UNINASSAU) - Campus Graças, Pernambuco, Brasil.

Hildeberto Dutra Sobral

e-mail hildebertosobral@gmail.com

\section{INTRODUÇÃO}

A fase de transição da infância para adolescência é bastante complexa, visto que durante o início da puberdade ocorrem várias mudanças tanto funcionais quanto morfológicas, algumas alterações que se pode notar são o pico de crescimento, tanto da estatura quanto dos órgãos sexuais. $\mathrm{Na}$ adolescência, essa mesclagem de maturação atrelada às experiências anteriores do indivíduo resulta em uma variação no desempenho motor (RÉ, 2011).

O TEA, também conhecido como Transtorno do Espectro do Autismo, afeta o indivíduo nos quesitos de socialização, comunicação e também se pode notar um comportamento repetitivo e incomum (LEVY, MANDELL, SCHULTZ, 2009). Alguns fatores neurológicos, genéticos e sociais podem afetar nos graus de severidade do autismo, porém a sua causa ainda é desconhecida. O TEA é caracterizado como um transtorno complexo, de caráter mental e que irá prejudicar o desenvolvimento neuro-motor do indivíduo, afetando assim na linguagem, interação social e cognição. Também se pode notar como característica marcante do TEA, principalmente na infância, os movimentos repetitivos e estereotipados (TORQUATO, COUTO, 2021).
Epidemiologicamente falando, uma em cada 160 crianças é autista e essa condição, que tende a aparecer na infância, persiste durante a adolescência e vida adulta. Os estudos também mostram que em 50 anos, ocorreu um aumento global na prevalência do TEA. Algumas explicações para esse aumento podem ser devido ao fato da maior conscientização acerca do tema, diagnósticos aprimorados para identificação e melhorias nas informações para a população. Em maio de 2014, na 67ํㅡㄹ Assembleia Mundial de Saúde, foi aprovada uma resolução que consistia em pontuar que a OMS, em parceria com os Estados Membros e agências parceiras no reforço das capacidades nacionais, colaborem para lidar com 0 Transtorno do Espectro do Autismo e outros problemas no desenvolvimento (GUEDES, TADA, 2015).

Existem três graus de autismo: o leve, o moderado e o severo. O leve poderá se observar esses sintomas citados no texto anteriormente, porém eles serão de uma forma mais branda, podendo assim o indivíduo viver uma vida com menos limitações, o moderado esses sintomas serão um pouco mais acentuados, necessitando assim de um cuidado maior e o severo atingirá bruscamente o adolescente, acarretando assim uma vida 
Judô e socialização

com maiores limitações e dependências (SILVA JUNIOR, 2012).

A UNESCO declarou o judô como o esporte mais indicado, pois além do esporte trabalhar o cognitivo, o social, o físico-motor, também é um excelente meio de desenvolvimento psicomotor do aluno, promovendo assim o seu bem estar tanto físico quanto psicológico (BATISTA., 2011).

\section{MÉTODO}

Trata-se de uma revisão integrativa da literatura acerca do perfil de Crianças e Adolescentes com o Transtorno do Espectro Autista (TEA), relacionada à saúde e melhora da qualidade de vida social e mental. A condução do presente estudo percorreu as seguintes etapas: pesquisa de artigos científicos realizada a partir das bases de dados eletrônicos, estabelecimento dos critérios de inclusão e exclusão, busca das referências, avaliação metodológica dos estudos incluídos, análise e síntese dos resultados. Essa pesquisa foi conduzida de acordo com Declaração PRISMA (Figura 1).

A questão norteadora dessa pesquisa foi: "Se a prática da modalidade Judô pode contribuir na melhora da coordenação, flexibilidade e qualidade de vida em adolescentes com Transtorno do Espectro Autista?". O levantamento bibliográfico ocorreu de março a maio de 2021, a partir dos trabalhos publicados e indexados, nas bases de dados eletrônicas Pubmed; Scielo; através
$O$ judô se torna benéfico para 0 adolescente com TEA devido ao fato de ter inúmeros benefícios, tais como: melhora da coordenação motora, descoberta de movimentos diferentes e novos para 0 indivíduo, melhora no domínio corporal, socialização, além de abordar também valores morais e éticos, já que o nome judô significa "caminho suave" e seu criador, Jigoro Kano, se baseou em 3 filosofias para criar a arte marcial: ceder para vencer, prosperidade e benefício mútuo e máxima eficácia do corpo e da mente (GONDIM, 2007).

do portal Biblioteca Virtual de Saúde BVS. Foram utilizados os operadores booleanos AND locados entre os descritores: Judô; Transtorno Espectro Autista; Adolescentes, Artes Marciais encontrados no Descritores em Ciência da Saúde (DeCS) no idioma português; e " Judô" "Transtorno Espectro Autista"; "Adolescentes", "Artes Marciais" em inglês e português.

Como critérios de inclusão: artigos de ensaios clínicos randomizados, nos idiomas inglês e português, publicado no período de 2016 a 2021 e que tinham como limite no estudo crianças e adolescente (10-19anos). E, utilizaram-se como critérios de exclusão: teses, dissertações, editoriais, estudos com animais e os que não respondiam à pergunta norteadora. Os artigos foram selecionados conforme respondessem o objetivo da pesquisa pela análise dos resumos e leitura na íntegra. 
Judô e socialização

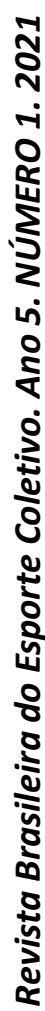

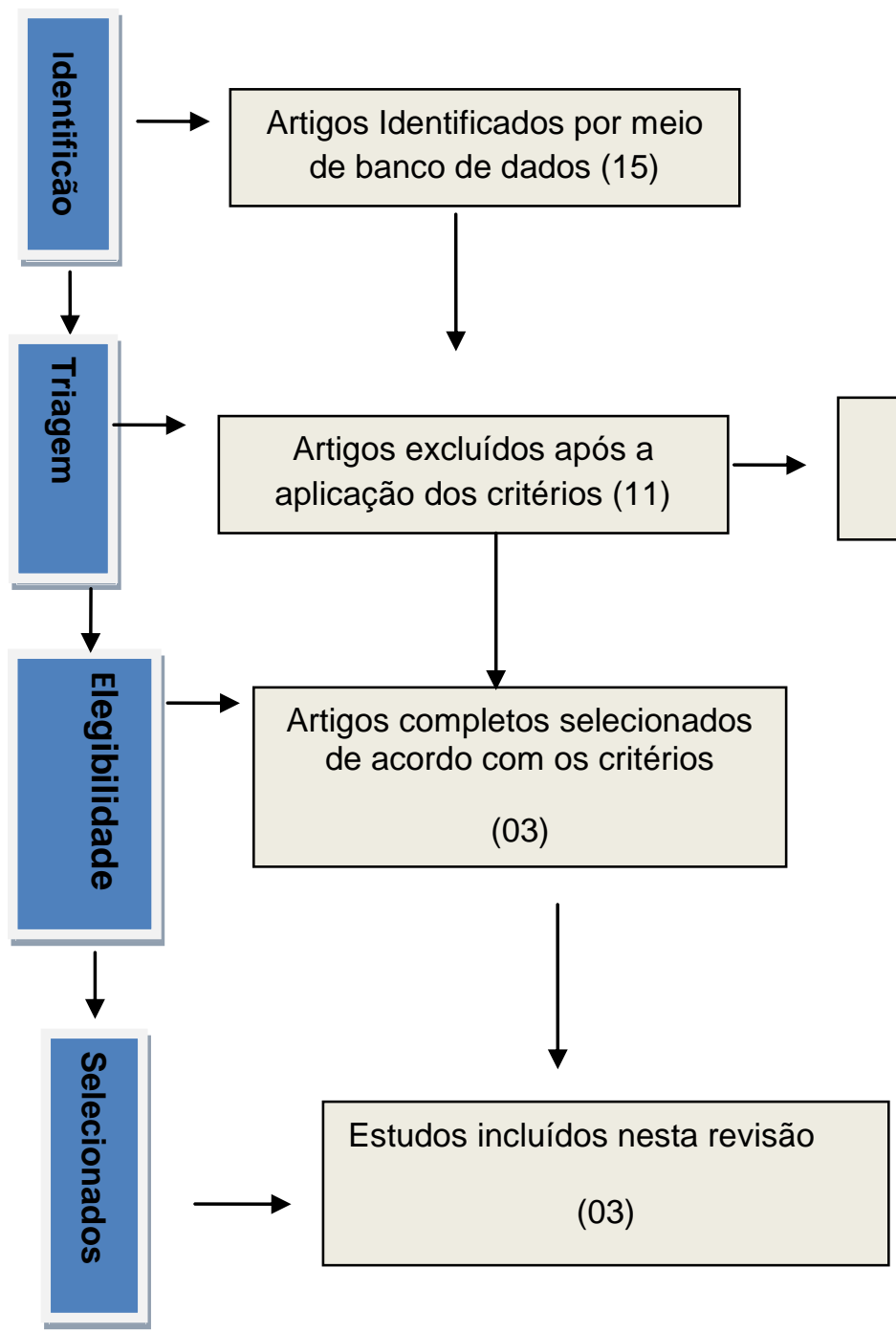

Figura 1 - Organograma dos estudos encontrados no referido estudo após o cheklist seguindo a declaração PRISMA

\section{RESULTADOS}

Tabela 1 - Resultados dos estudos encontrados após realizada a busca para referida pesquisa.

\begin{tabular}{|c|c|c|c|c|}
\hline $\begin{array}{l}\text { Autor } \\
\text { lano }\end{array}$ & Região & Objetivo do estudo & Instrumento utilizado & Principais Resultados \\
\hline $\begin{array}{l}\text { Rivera et } \\
\text { al., } \\
(2020)\end{array}$ & & $\begin{array}{l}\text { Analisar os efeitos e } \\
\text { fatores comportamentais } \\
\text { de crianças com TEA em } \\
\text { um programa de judô de } 8 \\
\text { semanas. }\end{array}$ & $\begin{array}{l}\text { Questionário/analise } \\
\text { quantitativas e qualitativas }\end{array}$ & $\begin{array}{l}\text { Resultados encontrados } \\
\text { através da entrevista com os } \\
\text { pais demonstram melhorias } \\
\text { perceptíveis em três temas } \\
\text { primários: interação social, } \\
\text { autoestima e diversão com o } \\
\text { programa. Também foi } \\
\text { observado, ao longo das } \\
\text { sessões de judô melhorias } \\
\text { comportamentais nesses }\end{array}$ \\
\hline
\end{tabular}


indivíduos.

Identificar a partir de casos, se o uso da atividade física em crianças com TEA chega a trazer benefícios

Sefen et al., (2020) em relação a manutenção da saúde.
Foi utilizado o programa TEACCH, mas com modificações para a recreação.
Os autores observaram que cada pessoa com TEA é afetada individualmente, com isso, 0 processo terapêutico realizado tem sua eficácia ao longo do programa. Os resultados trazem que os esportes e jogos aumentam as oportunidades daqueles indivíduos interagirem socialmente, melhorando assim habilidades de comunicação verbal e não verbal. Porém, ainda não há relatos evidentes de longo prazo.
Garcia et al., (2019)
Verificar em 8 semanas, quais efeitos a pratica do judô pode causar em jovens sedentários com TEA.
Foram utilizados análise estatística / Questionário demográfico acelerômetro Acti Graph
O resultado da amostra afirmou que os testes foram positivos, pois, nenhuma criança foi retirada do programa por comportamento e mais importante não houve a recusa de participação nas atividades, obtendo assim melhoras notáveis após a intervenção nas habilidades motoras e na socialização.

\section{DISCUSSÃO}

No estudo de Rivera et al., (2020) que observou 8 semanas da pratica do Judô para 25 jovens com TEA, com o intuito de examinar quais os efeitos o judô pode proporcionar a esses jovens; apesar dos resultados do estudo revelarem que não ocorrem mudanças significativas em relação ao comportamento relacionados ao Transtorno do Espectro Autista, nas entrevistas com os pais os resultados apontam para uma melhora nos aspectos da auto estima e habilidades sociais dos participantes.

Outro aspecto observado pelos pais positivamente nos participantes é que não existiu um comportamento de aversão quanto a pratica do judô ou um fator negativo em seus filhos quando praticaram o judô, apenas momentos de ansiedade para que a aula ocorresse, com exceção da primeira aula, pois os alunos ficavam ansiosos, o que acaba sendo normal, visto que é uma surpresa e quebra da rotina de vida dos mesmos, e a rotina diária é um fator importante para esses indivíduos (RIVERA, RENZIEHAUSEN, GARCIA, 2020).

O programa realizado por Sefen et al.,
(2020) para estudo com crianças e adolescentes com TEA, foi utilizado o método TEACCH (método onde é realizada uma avaliação para perceber os pontos fortes e de maior interesse assim como suas dificuldades, para a partir disso realizar uma estratégia individualizada); utiliza-se esse tipo de método para o tratamento e educação de crianças autistas e com deficiências relacionadas a comunicação. $O$ estudo mostrou que a atividades físicas como instrumento terapêutico tem seus benefícios para a saúde de crianças e adolescentes com TEA e a importância da relação dos pais nos encontros.

O autor ainda relata que cada pessoa com essa condição genética tem sintomas e 
características individualizadas, dando ênfase há uma carência em relação às pesquisas que as envolvam; efeitos de longo prazo com a atividade física se tratando de pessoas com o transtorno no espectro autista faz-se necessário se atentar a individualidade biológica de cada aluno e verificar efeitos crônicos do exercício como terapia para as crianças e adolescentes com o transtorno (SEFEN et al., 2020).

No estudo de Garcia et al., (2019), o projeto envolvia aplicação de atividade física moderada a vigorosa para reduzir o histórico de sedentarismo em jovens com TEA, aplicando programa de judô de 8 semanas. A eficácia preliminar no programa de judô com esses jovens diagnosticados com TEA, foi

\section{CONCLUSÃO}

Tendo em vista o aumento dos diagnósticos positivos do Transtorno do Espectro Autista, nos últimos anos, surge a necessidade de estudos mais aprofundados para conhecer os impactos que as comorbidades desse transtorno podem causar aos seus portadores.

Entretanto, através dos achados do referido estudo, observaram-se benefícios envolvendo a prática de atividades físicas e esportes, especificamente o judô, sobretudo na interação social e melhora das características cognitivas desses jovens. Outra questão observada foi que não apenas os pesquisadores chegaram a esse entendimento, mas os pais desses jovens durante o desenrolar dos estudos relataram a evolução dos seus filhos. alcançada com a porcentagem de seus benefícios de promoção à saúde, esses aumentaram em relação ao aumento da atividade física.

\begin{abstract}
Nas entrevistas com os pais, o interesse dos jovens em continuar o treinamento do judô após o fim do primeiro programa foi ressaltado, mas, devido à falta de tempo e deslocamento a participação foi menor e não a falta de interesse dos jovens, indicando assim que o judô pode ser utilizado como fator de promoção de saúde e de qualidade de vida para esse público. O autor afirma ainda que novos estudos são necessários, pois, a amostra foi limitada (GARCIA et al., 2020).
\end{abstract}

Ao logo do nosso estudo observou-se que os autores relataram que não houve uma evolução considerada tão eficaz nos adolescentes com TEA que participaram das pesquisas, com os instrumentos utilizados, porem também não foram vistos fatores que contribuíssem para uma piora por meio das atividades atribuídas aos jovens com TEA.

Contudo, em virtude da complexidade e falta de estudos em longo prazo, relacionados ao Transtorno do Espectro Autista (TEA), faz se necessário à busca por mais pesquisas e terapias tanto para os profissionais de saúde quanto para as famílias, com intuito de amenizar e estimular 0 desenvolvimento social de maneira mais natural.

\section{REFERÊNCIAS}

BATISTA, M. A. S.; SIXTO, Cubo. La práctica de judo en relacion con el autoconcepto, la autoestima y el rendimento escolar de los estudiantes de primer ciclo de primaria. 2011. Tese (Doutorado) - Polytechnic Institute of Castelo Branco - Castelo Branco, Portugal. Disponível em: DOI:10.13140/RG.2.1.4608.2007. Acesso em: 22/04/2021.

GARCIA, J. M. et al. Brief Report: Preliminary Efficacy of a Judo Program to Promote Participation in Physical Activity in Youth with Autism Spectrum Disorder. Journal of Autism and Developmental Disorders, [s. l.], v. 50, n. 4, p. 1418-1424, 2020. Disponível em: https://doi.org/10.1007/s10803-01904338-w. Acesso em: 17/04/2021

GONDIM, D. F. Aspectos metodológicos aplicados ao ensino do judô para crianças. Or: Prof. Ms. Marco Aurélio Oliveira (ESEF-UPE), [s. I.], p. 1-26, 2007. Disponível em: http://www.Inj.com.br/monografia_denis_2012pdf.pdf. Acesso em: 20/03/2021

GUEDES, N. P. S.; TADA, I. N. C. A produção científica brasileira sobre autismo na psicologia e na educação. Psicologia: Teoria e Pesquisa, [s. I.], v. 31, n. 3, p. 303-309, 2015. Disponível em: https://doi.org/10.1590/0102-37722015032188303309. Acesso em: 30/04/2021 
Judô e socialização

LEVY, S. E.; MANDELL, D. S.; SCHULTZ, R. T. Autism. The Lancet, [s. I.], v. 374, n. 9701, p. 16271638, 2009. Disponível em: https://doi.org/10.1016/S0140-6736(09)61376-3. Acesso em: 22/04/2021

RÉ, A. H. N. Crescimento, maturação e desenvolvimento na infância e adolescência: Implicações para o esporte. Motricidade, [s. I.], v. 7, n. 3, p. 55-67, 2011. Disponível em: https://doi.org/10.6063/motricidade.7(3).103. Acesso em: 30/04/2021

RIVERA, P.; RENZIEHAUSEN, J.; GARCIA, J. M. Effects of an 8-Week Judo Program on Behaviors in Children with Autism Spectrum Disorder: A Mixed-Methods Approach. Child Psychiatry and Human Development, [s. I.], v. 51, n. 5, p. 734-741, 2020. Disponível em: https://doi.org/10.1007/s10578020-00994-7. Acesso em: 17/04/2021

SEFEN, Jessica Atef Nassef et al. Beneficial Use and Potential Effectiveness of Physical Activity in Managing Autism Spectrum Disorder. Frontiers in Behavioral Neuroscience, [s. I.], v. 14, n. October, p. 1-8, 2020. Disponível em:https://doi.org/10.3389/fnbeh.2020.587560. Acesso em: 22/04/2021.

SILVA JUNIOR, L. P. Avaliação do perfil motor de crianças autistas de 7 a 14 anos freqüentadoras da Clínica Somar da Cidade de Recife - PE. [s. I.], p. 73, 2012. Disponível em: http://dspace.bc.uepb.edu.br/jspui/handle/123456789/761. Acesso em: 30/04/2021.

TORQUATO, E.; COUTO, C. R. Efeitos da prática do judô na coordenação motora de crianças e adolescentes autistas effects of judo practice on the motor coordination. [s. I.], p. 3-14, 2021. Disponível em: https://doi.org/10.36311/2674-8681.2021.v22n1.p3-14. Acesso em: 17/04/2021. 